\title{
Mastectomía ahorradora die piel tipo IV con injerto libre de areola-pezón para reconstrucción con prótesis en cirugia reductora de riesgo
}

\section{Reductive risk surgery with prosthetic reconstruction by using type IV skin sparrow mastectomy with riee nipple-areola graft}

\author{
E. Mauricio AVELLANEDA OVIEDO*, Alejandra GARCÍA NOVOA*, Pablo PALACIOS GARCÍA ${ }^{\star \star \star ~}$ \\ F. Javier PACHECO COMPAÑA*, Benigno ACEA NEBRIL**, Luis ALBAINA LATORRE ${ }^{* *}$
}

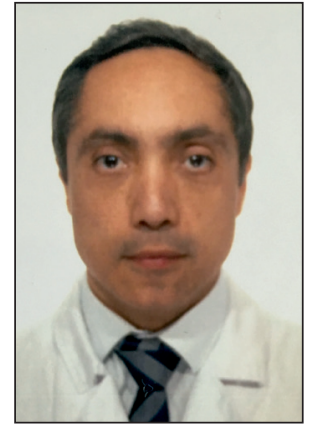

Avellaneda Oviedo E.M

Resumen

Abstract

Introducción y Objetivo. Los avances en el tratamiento del cáncer de mama y las múltiples opciones reconstructivas para las pacientes hacen necesario entender una variedad de procedimientos a diferentes niveles según el tipo de cirugía oncológica indicada en cada caso: desde reconstrucción diferida postmastectomía con expansores de piel y segundo tiempo para implantación de prótesis definitiva, hasta reconstrucción inmediata con técnicas complejas de Microcirugía en un solo tiempo.

Ilustramos paso por paso una técnica de reconstrucción mamaria inmediata en un solo tiempo utilizando prótesis aloplásticas tras mastectomía ahorradora de piel en pacientes subsidiarias de cirugía reductora de riesgo, antes llamada profiláctica, que es una indicación relativamente frecuente en nuestro medio a fin de hacer prevención primaria y secundaria del cáncer de mama.

Material y método. Descripción detallada de la técnica quirúrgica de mastectomía tipo IV con injerto libre de complejo areola-pezón (MIVAP), ejemplificando con dibujos y fotos didácticas para su entendimiento y presentando 9 casos con sus diferentes indicaciones.

Resultados. La indicación más frecuente en nuestra muestra fue el antecedente de cáncer ginecológico (ovario o mama) asociado a mutación en el gen BRCA 1 o 2. Otras indicaciones fueron: positividad para mutación del BRCA 1 o 2 sin antecedentes tumorales, mutaciones en el gen supresor p53 (síndrome de Li-Fraumeni), cancerofobia o alta carga familiar de cáncer de mama u ovario (más de 3 familiares en primera línea) sin positividad para ninguna mutación genética.

Tuvimos 1 caso de infección postquirúrgica, 1 linfedema postquirúrgico grado II en biopsia selectiva de ganglio centinela (BSGC) y 1 necrosis parcial de complejo areola pezón.

El efecto estético negativamente valorado más frecuente fue la fibrosis tisular con falta de volumen en los cuadrantes mediales de la mama (imagen ancha del escote). La reincorporación a la vida habitual tuvo una media de 34 días. El tipo de prótesis más utilizado fue texturizada de alta proyección, de entre 250 y 350 gr.

Conclusiones. La MIVAP es una técnica válida para reconstrucción mamaria en pacientes subsidiarias de mastectomía reductora de riesgo cuando la indicación es correcta. Aunque con ciertas limitaciones estéticas, se realiza en un solo tiempo y minimiza las complicaciones del uso de expansores. Los casos presentados evolucionaron favorablemente, con baja incidencia de complicaciones y elevado grado de satisfacción.

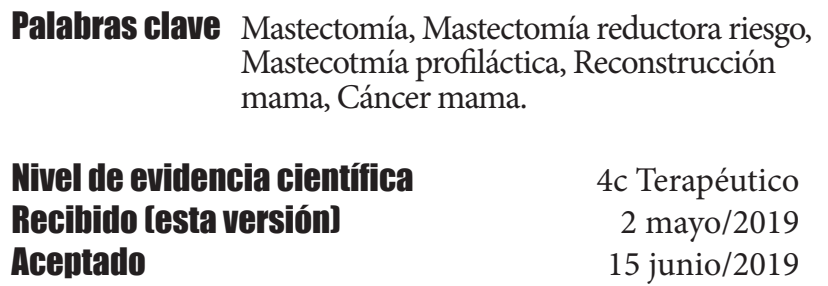

Background and objective. Advances in treatment of breast cancer and different reconstructive options make it necessary to understand a variety of procedures depending on the type of oncological surgery performed on each patient. The range of reconstructive procedures varies from delayed post-mastectomy reconstruction, using skin expanders and in a second time implantation of a definitive prosthesis, to immediate reconstruction by complex microsurgical techniques in a single time.

Our aim is to illustrate, step by step, an immediate breast reconstruction technique in a single time using alloplastic prostheses after a skin-sparing mastectomy in patients that are subsidiary to risk-reducing surgery, formerly called prophylactic mastectomy, is a frequent indication in our setting used for primary and secondary prevention of breast cancer.

Methods. The procedure of a type IV mastectomy with nipple-areola complex free graft (MIVAP) is carefully described. Original drawings and didatic images are shown for the correct understanding of the procedure. We present 9 cases with their different indications.

Results. The most frequent indication in our sample to perform a MIVAP was a history of gynecological cancer (ovary or breast) associated with a mutation in BRCA 1 or 2 gene. Other indications were: positivity for BRCA 1 or 2 mutation without cancer history, mutations in p53 suppressor gene (Li-Fraumeni syndrome), cancer-phobia or high family burden of breast or ovarian cancer (more than 3 first consanguinity line parents) without any mutation.

There was a case of postoperative infection, 1 patient developed grade II postsurgical lymphedema associated with selective sentinel lymph node biopsy (BSGC), and 1 suffered nipple-areola partial necrosis.

The most frequent negatively aesthetic effect was tissue fibrosis with lack of volume in the medial quadrants of the breast (broad image of the neckline). Patients returned to the habitual life around 34 days after the procedure. The prosthesis most frequently used was textured, high projection between 250 and $350 \mathrm{gr}$.

Conclusions. MIVAP is a valid technique for breast reconstruction in candidates for risk-reducing mastectomy. Although there are certain aesthetic limitations, it is performed in a single surgical time and avoids complications related to cutaneous expanders. Our cases evolved favorably, with a low incidence of complications and a high degree of satisfaction.

Key words Mastectomy, Risk reduction mastectomy, breast reconstruction, Breast cancer.

\section{Level of evidence Received [this version] Acceptei} 4c Therapeutic $2 \mathrm{May} / 2019$

Conflicto de intereses: Los autores declaran no tener ningún interés financiero relacionado con el contenido de este artículo.

Financiación: No hubo fuentes externas de financiación para este trabajo.

\footnotetext{
Cirujano Plástico, Médico Adjunto, Servicio de Cirugía Plástica

** Cirujano General, Médico Adjunto, Servicio de Cirugía General y del Aparato Digestivo

*** Médico Interno Residente, Servicio de Cirugía Plástica

Complejo Hospitalario Universitario de La Coruña, La Coruña, España
} 
Introduccion

El mejor entendimiento actual del cáncer de mama, los avances en las técnicas quirúrgicas y la optimización de los métodos adyuvantes han permitido que la cirugía conservadora sea considerada como la primera línea de tratamiento. Aunque una mastectomía clásica es inevitable en pacientes con tumores de gran tamaño, multicéntricos o con contraindicación para radioterapia postoperatoria, la tendencia actual apunta hacia utilizar técnicas que conserven la mayor cantidad de tejido autólogo sin empeorar el pronóstico general de la paciente.

Los avances en el diagnóstico genético y los modernos métodos radiológicos han permitido que las indicaciones de las técnicas profilácticas hayan aumentado considerablemente. Estos procedimientos suelen plantearse a personas sanas, sin antecedentes oncológicos, pero con alto riesgo de desarrollar un tumor; también a pacientes mastectomizadas por causa de un cáncer mamario para reducir el riesgo en la mama contralateral. ${ }^{(1)}$

La mastectomía reductora de riesgo, también conocida como mastectomía profiláctica, consiste en extirpar la mayor cantidad de tejido posible de una mama sana para reducir el riesgo potencial de desarrollar cáncer. Está indicada en pacientes que tienen mayor riesgo de desarrollar cáncer de mama que el resto de la población. Sin embargo no se puede decir que elimine el riesgo de padecerlo, únicamente que lo reduce.

El Complejo Hospitalario Universitario de La Coruña es una institución pública de cuarto nivel y el centro principal de referencia de la provincia de La Coruña en la Comunidad Autónoma de Galicia (España). Dentro de esta institución funciona la Unidad de Senología y Patología Mamaria, en la que se realizan un promedio aproximado de 500 cirugías al año relacionadas con dicho órgano. Se tratan pacientes con enfermedad oncológica de la mama, patología benigna y cirugía reconstructiva. La Unidad está formada por especialistas de los Servicios de Cirugía Plástica, Cirugía General, Ginecología, Radiología, Oncología y Radioterapia.

Los especialistas en Cirugía General y Ginecología proporcionan el tratamiento inicial de la patología benigna y oncológica en estrecho contacto con los especialistas en Cirugía Plástica, quienes se encargan del proceso reconstructivo cuando este es necesario. Dicho proceso puede incluir la simetrización de la mama contralateral (reducción y pexia), la colocación de prótesis o expansores mamarios, la realización de colgajos pediculados o libres, la remodelación de cirugías previas o la reconstrucción primaria en la mastectomía reductora de riesgo (MRR). También se encarga del tratamiento de las pacientes con gigantomastia, realizando reducciones mamarias bilaterales no relacionadas con procesos oncológicos. No contempla la realización de Cirugía Estética de la mama y únicamente se realizan tratamientos incluidos en el plan de la Seguridad Social española (sistema público nacional de salud).

Este trabajo pretende revisar la técnica quirúrgica de mastectomía tipo IV con injerto libre de complejo areola pezón (MIVAP), presentando la experiencia de la Unidad de Cirugía de la Mama (UMA) de nuestro hospital mediante una serie de casos seleccionados de pacientes con alto riesgo de desarrollar un cáncer mamario en las que empleamos esta técnica como alternativa reconstructiva inmediata con prótesis en un solo tiempo quirúrgico.

\section{Material y método}

Exponemos la técnica quirúrgica y analizamos 9 casos de mujeres con indicación de mastectomía ahorradora de piel y/o MRR a quienes se les realizó una MIVAP. El rango de edad de las pacientes fue de entre 24 y 45 años.

Incluimos mujeres que optaron por una mastectomía profiláctica primaria y también pacientes que solicitaron una mastectomía profiláctica secundaria: antecedentes de cáncer mamario, que habían sido curadas mediante cualquier tipo de intervención previa diferente a una mastectomía, como por ejemplo una cuadrantectomía o una oncoplástia. El 50\% de las pacientes de la muestra no había padecido ningún cáncer y por lo tanto se trataba de mujeres sanas.

Excluimos pacientes con otro tipo de mastectomía diferente a la MIVAP o con cualquier otra indicación para la mastectomía diferente a la opción de reducción de riesgo.

Revisamos las diferentes complicaciones posibles tras la intevención: seroma, hematoma, infección, exposición de la prótesis, dehiscencia de la herida quirúrgica, linfedema, si el objetivo estético fue o no alcanzado y el tiempo medio de reincorporación a su vida habitual, y comparamos con las existentes en la literatura revisada.

Para evaluar el grado de satisfacción global de las pacientes y de los cirujanos con el resultado obtenido tras la intervención, plateamos un cuestionario anónimo a cada una de las pacientes con las siguientes preguntas a los 6 meses y al año de la intervención:

1.- ¿Ha tenido algún problema para reincorporarse a su trabajo una vez transcurrido 1 mes después de la intervención?

2.- ¿Resultó incomodo o doloroso el periodo postoperatorio inmediato (primera semana tras la intervención). Marque del 0 al 10 el grado de incomodidad o 
dolor que pudo presentar. Marque con una X en una escala analógica visual, donde crea que corresponde: 0 se considera nada de dolor o molestia tras la intervención y 10 si tuvo dolor o molestia insoportable.

3.- ¿Se siente más tranquila después de la cirugía respecto a la posibilidad latente de desarrollar un cáncer de mama?

4.- ¿La cirugía ha afectado de alguna manera la percepción propia de su imagen corporal? ¿Ha necesitado algún tipo de ayuda externa (psicólogo o psiquiatra)?

5.- Valore de 0 a 10 el grado de satisfacción respecto al cumplimiento de sus expectativas estéticas después de la cirugía.

A los cirujanos se les aplicó el siguiente cuestionario también 6 meses y 1 año después de la intervención:

1.- ¿Cree que su paciente está satisfecha con el resultado?

2.- ¿Ha tenido alguna complicación tras la intervención quirúrgica?

3.- ¿Estéticamente, cuál es su grado de satisfacción con la intervención realizada en una escala de 0 a 10 donde 0 es nada satisfecho y 10 totalmente satisfecho?

Presentamos la técnica quirúrgica empleada y recogemos nuestra casuística.

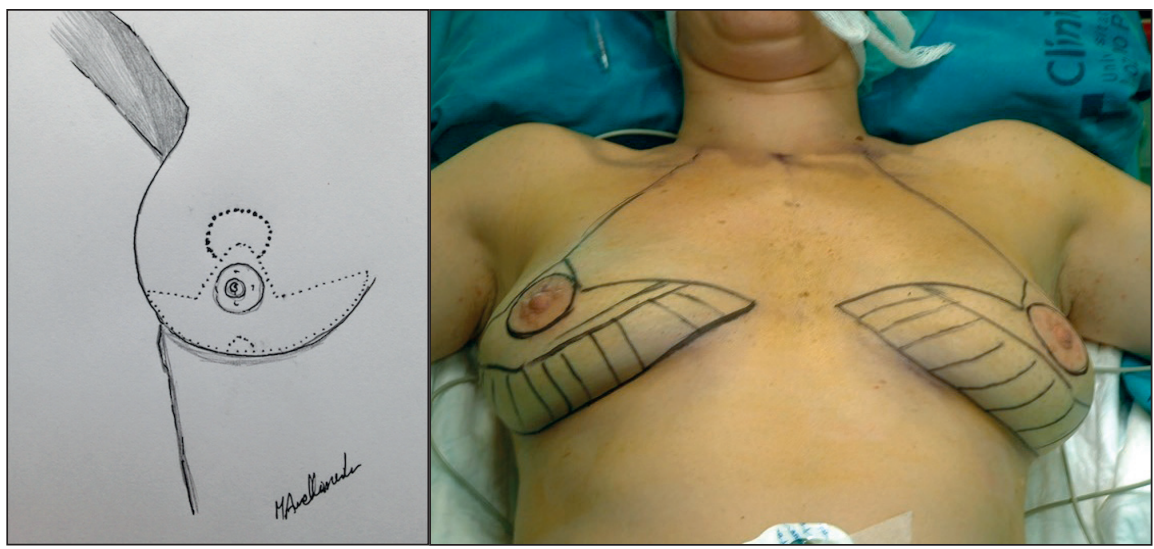

Figura 1. A Patrón de reducción mamaria de Wise. B. Patrón de Wise dibujado en la piel de la paciente en la que se conservará un colgajo dermograso desepidermizado en el polo inferior (zona rayada) para dar cobertura al implante.

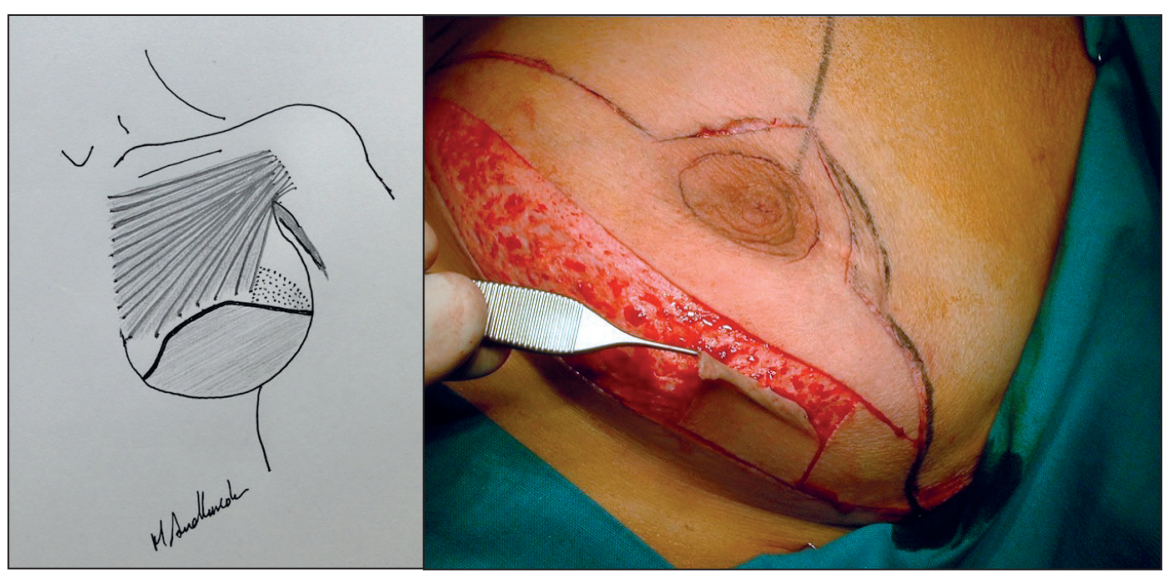

Figura 2. Diseño de colgajo dermograso para cubrir el implante en los polos inferiores. A. Rayado: músculo pectoral mayor; sombreado: colgajo dermograso; Punteado: prótesis mamaria. B. Desepidermización del colgajo dermograso.

\section{Técnica quirúrgica}

La MIVAP consiste en una mastectomía con fines profilácticos para reducir el riesgo oncológico empleando el patrón de reducción mamaria de Wise. En la Fig. 1 ilustramos el diseño de las incisiones, conservando un colgajo dermo-graso desepidermizado en los cuadrantes inferiores para dar cobertura al implante (Fig. 2). El colgajo dermo-graso obtenido a partir del polo inferior de la mama tras su desepidermización se afronta mediante sutura al músculo pectoral mayor desinsertado en su borde inferior, proporcionando entre ambos un bolsillo adecuado para la cobertura del implante (Fig. 3).

Pesamos el tejido mamario en una báscula digital y lo enviamos para su estudio anatomopatológico. Realizamos la biopsia selectiva del ganglio centinela (BSGC) en los casos en los que haya datos clínicos o radiológicos de cualquier posible afectación tumoral de la mama a extirpar (incluimos para la valoración la región de la axila), como por ejemplo la presencia de tumoraciones palpables o microcalcificaciones en la mamografía que de forma preoperatoria, se realiza protocolariamente a todas las pacientes. Para el abordaje de la BSGC utilizamos la misma incisión de la mastectomía.

Una vez realizada la extirpación de la piel, grasa y glándula mamaria por encima del músculo pectoral, levantamos dicho músculo por su borde inferior y disecamos un bolsillo entre este y la pared costal, con el tamaño adecuado para alojar el implante escogido. Posteriormente colocamos la prótesis en dicho espacio y cerramos la cavidad con suturas reabsorbibles que afrontan el borde inferior del músculo pectoral con el colgajo dermo-graso desepidermizado, cubriendo por completo el implante. Finalmente procedemos al cierre de la piel siguiendo el patrón de Wise, cubriendo todo el montaje subcutáneo, y llevamos a cabo el injerto libre de areola y pezón (Fig. 4).

\section{Resultados}

La muestra estuvo compuesta por 9 pacientes que acudieron a nuestra Unidad de Patología Mamaria durante un periodo de 2 años solicitando la realización de una mastectomía reductora de riesgo con al menos una indicación adecuada para la misma, $y$ en consecuencia fueron sometidas 

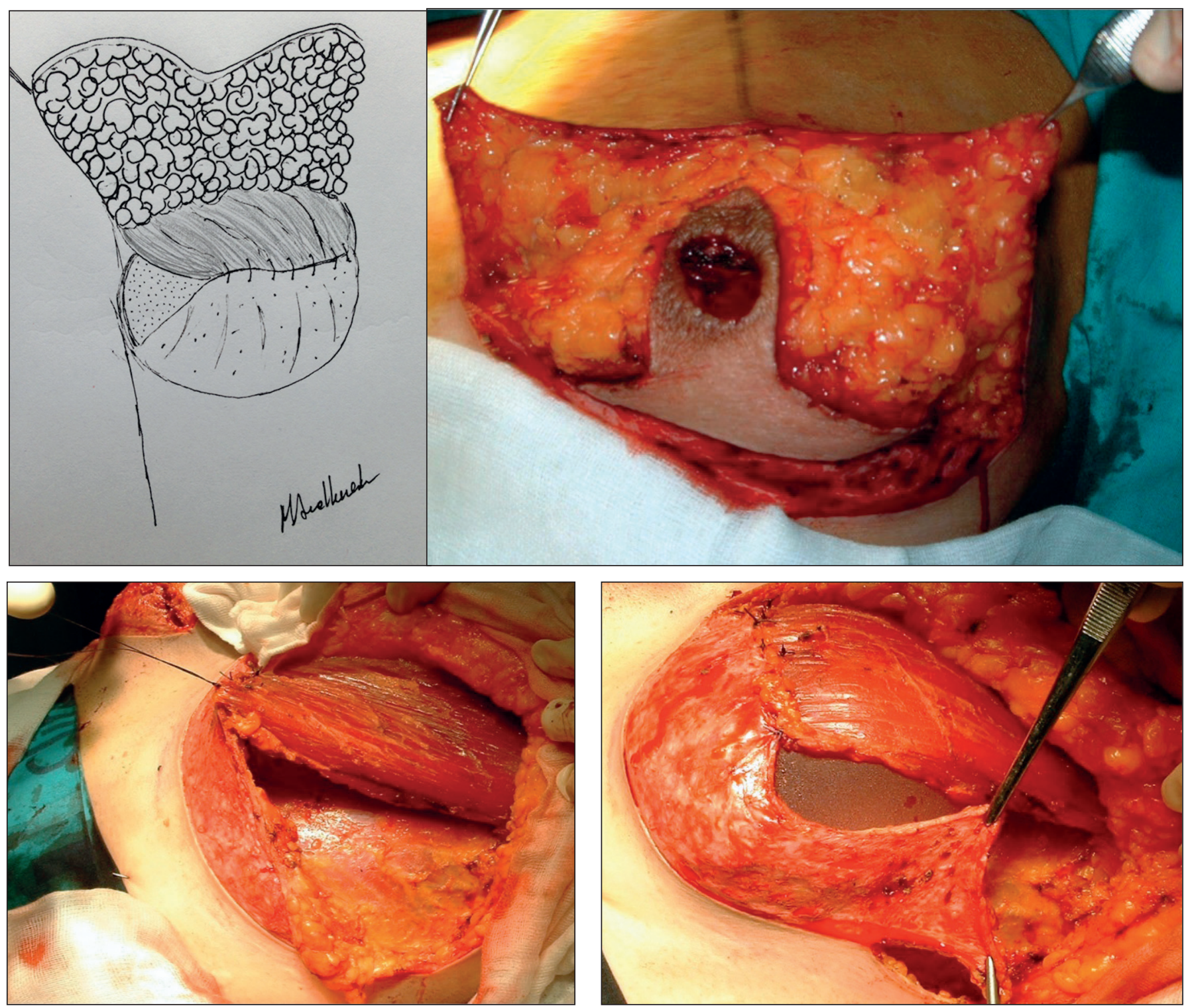

Figura 3. Prótesis dentro del bolsillo formado por el músculo pectoral mayor y el colgajo dermograso.

a tratamiento quirúrgico en dicha Unidad. La media de edad fue de 35.8 años (mínima de 24 y máxima de 45 años). Todas fueron mujeres.

La indicación más frecuente para realizar una MIVAP fue el antecedente de cáncer ginecológico (ovario o mama) asociado a una mutación en el gen BRCA 1 o BRCA 2 ( 3 pacientes). También se indicó en 2 pacientes con positividad para mutación del BRCA 1 o BRCA 2, sin antecedentes tumorales. Solo tuvimos 1 caso con mutaciones en el gen supresor p53 (síndrome de Li-Fraumeni), 1 caso de alta carga familiar de cáncer de mama u ovario (más de 3 familiares en primera línea) pero sin positividad para ninguna mutación genética, $\mathrm{y}$ 2 pacientes con cancerofobia completaron el resto de la muestra.

En la tabla I resumimos las indicaciones de las pacientes del grupo de estudio.

Los implantes utilizados en todos los casos fueron de gel cohesivo de silicona, anatómicos, texturizados de alta proyección y con una media de volumen de entre 250 a $350 \mathrm{cc}$.

Durante la evaluación de las complicaciones encontramos que no se presentaron seromas ni hematomas, pero si 1 caso de infección postoperatoria que hizo necesaria la retirada de la prótesis. No identificamos casos de dehiscencias de sutura, ulceración de la piel por efecto de la prótesis o exposición espontanea del implante. Realizamos BSGC en 3 pacientes por la presencia de microcalcificaciones subclínicas en la mamografía previa a la cirugía: 2 pacientes con antecedente de cáncer ginecológico (ovario o mama) asociado a una mutación en el gen BRCA 1 o 2 y 1 con positividad para mutación del BRCA 1 o 2 pero sin antecedentes tumorales. Una de estas 3 pacientes, con antecedente de cáncer ginecológico (mama) asociado a una mutación en el gen BRCA 2, desarrolló linfedema estadio IIa a los 6 meses de la cirugía (valoración según el estadiaje de los linfedemas de la International Society of Lymphology) (Tabla II). ${ }^{(2)}$ 

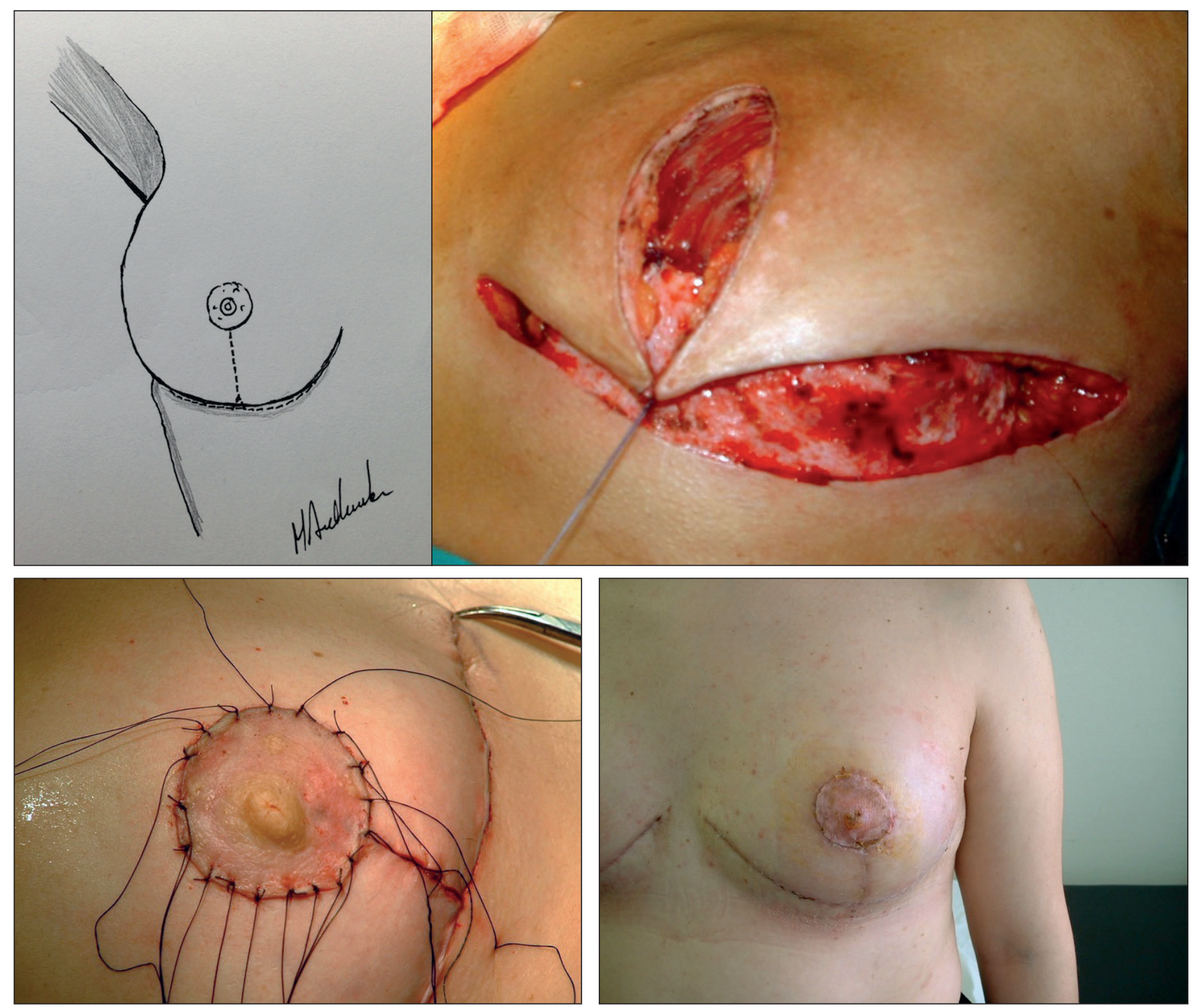

Figura 4. A y B. Finalización del procedimiento. C. Injerto libre de areola y pezón. D. Aspecto a las 2 semanas tras la cirugía.

Con respecto al cuestionario que llevamos a cabo entre las pacientes, ninguna de ellas presentó problemas para incorporarse a su trabajo pese a las complicaciones encontradas (Tabla I). Tres pacientes (33\%) indicaron que el periodo postoperatorio fue doloroso y $6(66 \%)$ que fue molesto y poco doloroso. Objetivamente, la media muestral del dolor/molestia en la escala análoga visual fue de 4.3. Cuando se les preguntó si estaban más tranquilas después de la cirugía respecto a la posibilidad latente de desarrollar un cáncer de mama, 9 pacientes (100\%) respondieron afirmativamente. Ninguna necesitó ayuda psicológica y solo $2(22 \%)$ indicaron que les gustaba más la apariencia de su pecho antes de la intervención. La media de satisfacción con el cumplimiento de las expectativas estéticas tras la intervención se situó en 7.4 en la escala análoga visual aplicada para esta pregunta.

Cuatro cirujanos del equipo valoraron a todas las pacientes en consultas de revisión mensuales realizadas independientemente a cada una de ellas. Según los datos recogidos, todas las pacientes se encontraban satisfechas con el objetivo de la intervención (reducción de riesgo de cáncer de mama), pero 2 indicaron que el resultado estético había sido inferior al esperado. Teniendo en cuenta las limitaciones estéticas y habiendo cumplido con el objetivo de la intervención (reducción de riesgo) la media de satisfacción de los cirujanos se situó en un 8.5 en la escala análoga visual. Los 4 cirujanos coincidieron en que 8 de las 9 pacientes el resultado estético en cuanto a cicatrices y forma de la mama era satisfactorio, pero en 1 de ellas el resultado estético era poco satisfactorio debido a un aspecto vacío de los polos mediales de la mama y una cicatriz en el complejo areola pezón, resultante de una necrosis parcial del complejo areola pezón que se había corregido mediante tatuaje.

La reincorporación a la vida diaria ocurrió alrededor de los 34 días (mínima de 27 y máxima de 45 días). Las pacientes jóvenes tardaron más tiempo en reanudar su vida habitual que las de mayor edad. 
Tabla I. Indicaciones de la técnica en las pacientes de nuestro grupo de estudio

\begin{tabular}{|l|c|}
\hline \multicolumn{1}{|c|}{ Indicaciones } & Casos \\
\hline Antecedente de cáncer ginecológico (ovario o seno) asociado a una mutación en el gen BRCA 1 o 2 & 3 \\
\hline Positividad para mutación del BRCA 1 o 2 sin antecedentes tumorales & 2 \\
\hline Mutaciones en el gen supresor p53 (síndrome de Li-Fraumeni) & 1 \\
\hline Cancerofobia & 2 \\
\hline Alta carga familiar de cáncer de mama u ovario, sin positividad para ninguna mutación genética. & 1 \\
\hline TOTAL & 9 \\
\hline
\end{tabular}

TABLA II. Complicaciones registradas en las pacientes de nuestro grupo de estudio

\begin{tabular}{|l|c|c|c|c|}
\hline \multicolumn{1}{|c|}{ Complicaciones } & Casos & $\begin{array}{c}\text { Implante } \\
\text { (cc) }\end{array}$ & Proyección & $\%$ \\
\hline Infección postoperatoria & 1 & 330 & alta & 11 \\
\hline Linfedema & 1 & 295 & alta & 11 \\
\hline Necrosis parcial del complejo areola-pezón & 1 & 265 & alta & 11 \\
\hline Fibrosis con falta de volumen medial & 9 & & & 100 \\
\hline Pobre resultado estético percibido par la paciente & 2 & 330 & alta & 22 \\
\hline Pobre resultado estético percibido por los cirujanos & 1 & 265 & alta & 11 \\
\hline
\end{tabular}

A continuación presentamos en imágenes y con leyenda explicativa 6 casos de nuestra muestra que consideramos podrían ilustrar claramente las diferentes indicaciones por las que llevamos a cabo una MIVAT, a saber:

Caso 1. Carcinoma ductal infiltrante in situ en mama derecha + BRCA 2 (Fig. 5).

Caso 2. Antecedente de carcinoma seroso de ovario bilateral + BRACA 1 (Fig. 6) .

Caso 3. Carcinoma ductal infiltrante in situ en mama izquierda + BRCA 2 (Fig. 7).

Caso 4. Cancerofobia (Fig. 8).

Caso 5. Antecedentes familiares (Fig. 9).

Caso 6. Síndrome de Li Fraumeni (Fig. 10).

\section{Discusión}

Las cirugías agresivas y mutilantes y/o que impliquen una gran distorsión en la imagen corporal como la mastectomía radical, asocian una importante carga psicológica para las pacientes que las padecen pudiendo desembocar en una depresión. ${ }^{(3)}$ El cáncer de mama representa una patología con elevada inciden- cia a mundial, registrándose más de 1.676.000 nuevos casos al año, siendo el cáncer más frecuente entre las mujeres. ${ }^{(4)}$

Históricamente la finalidad del tratamiento ha sido oncológica radical, extendiendo las amputaciones desde el centro del tórax hasta la axila. Sin embargo, durante los últimos años el avance en el tratamiento quirúrgico conservador para el cáncer de mama con técnicas como la mastectomía subtotal, la cuadrantectomía, la oncoplastia o la linfadenectomía selectiva, etc., de la mano de nuevos tratamientos médicos adyuvantes como la quimioterapia, los medicamentos biológicos, la quimioterapia neoadyuvante, la radioterapia, etc., han permitido a la cirugía realizar tratamientos cada vez más conservadores, proporcionando a estas pacientes ciertas ventajas tanto físicas como psicológicas.

Un paso más adelante en la prevención del cáncer de mama es la realización de una mastectomía reductora de riesgo (MRR), mal llamada hasta hace unos años mastectomía profiláctica puesto que no evita la aparición del cáncer de mama sino que solo disminuye su riesgo, en pacientes sanas con alto riesgo de sufrir un cáncer o que habiendo sido curadas de uno deciden reducir el riesgo de presentarlo en la mama contralateral. 

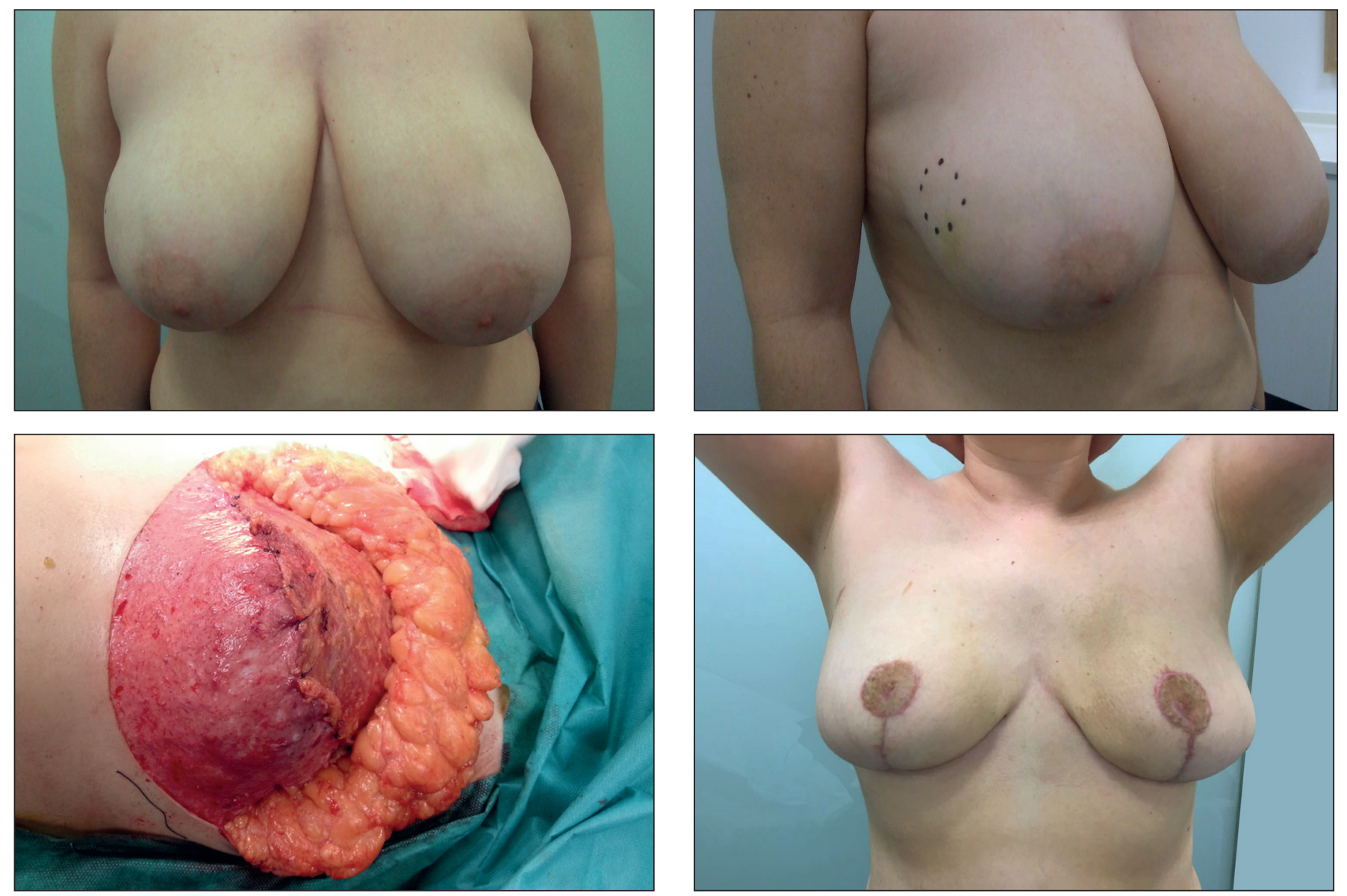

Figura 5. Caso 1. Indicación: carcinoma ductal infiltrante in situ en mama derecha + BRCA 2. Paciente de 45 años. A y B. Preoperatorio. El círculo punteado es la ubicación del tumor. C. Bolsillo introperatorio. D. Postoperatorio a las 3 semanas. Implante anatómico de gel cohesivo de silicona de 330 cc.
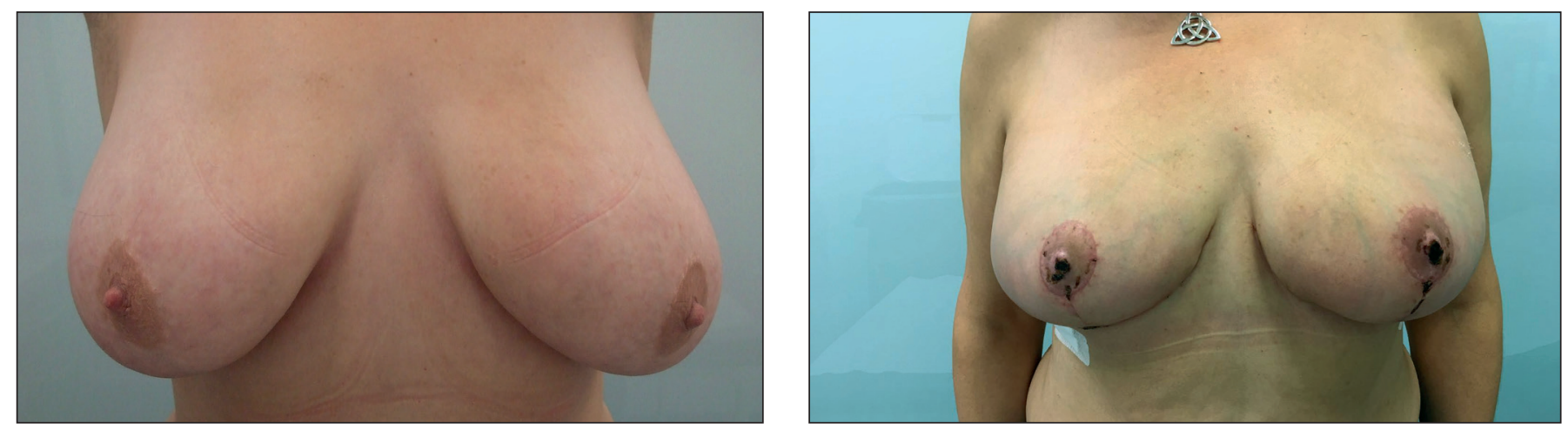

Figura 6. Caso 2. Indicación: antecedente de carcinoma seroso de ovario bilateral + BRACA 1. Paciente de 37 años. A. Preoperatorio. B. Postoperatorio a los 10 días. Implante anatómico de gel cohesivo de silicona de $250 \mathrm{cc}$.
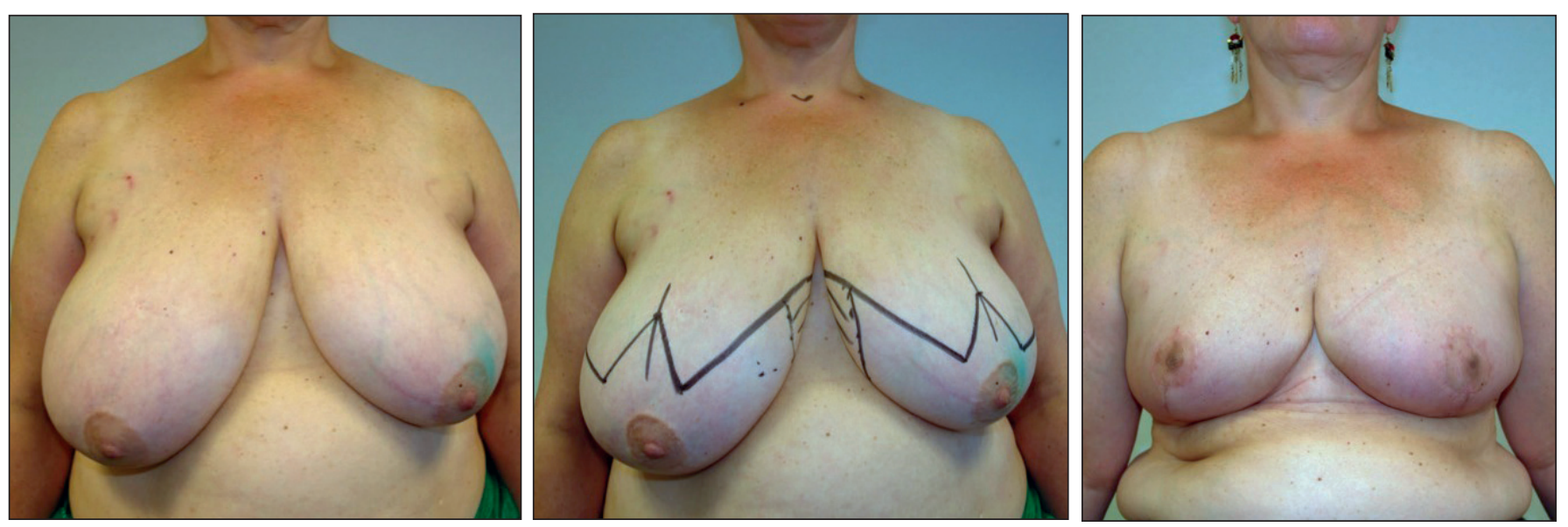

Figura 7. Caso 3. Indicación: carcinoma ductal infiltrante in situ en mama izquierda + BRCA 2. Paciente de 43 años con cáncer en mama izquierda e importante asimetría mamaria. A y B. Preoperatorio. C. Postoperatorio al mes. Implante anatómico de gel cohesivo de silicona de $325 \mathrm{cc}$. 

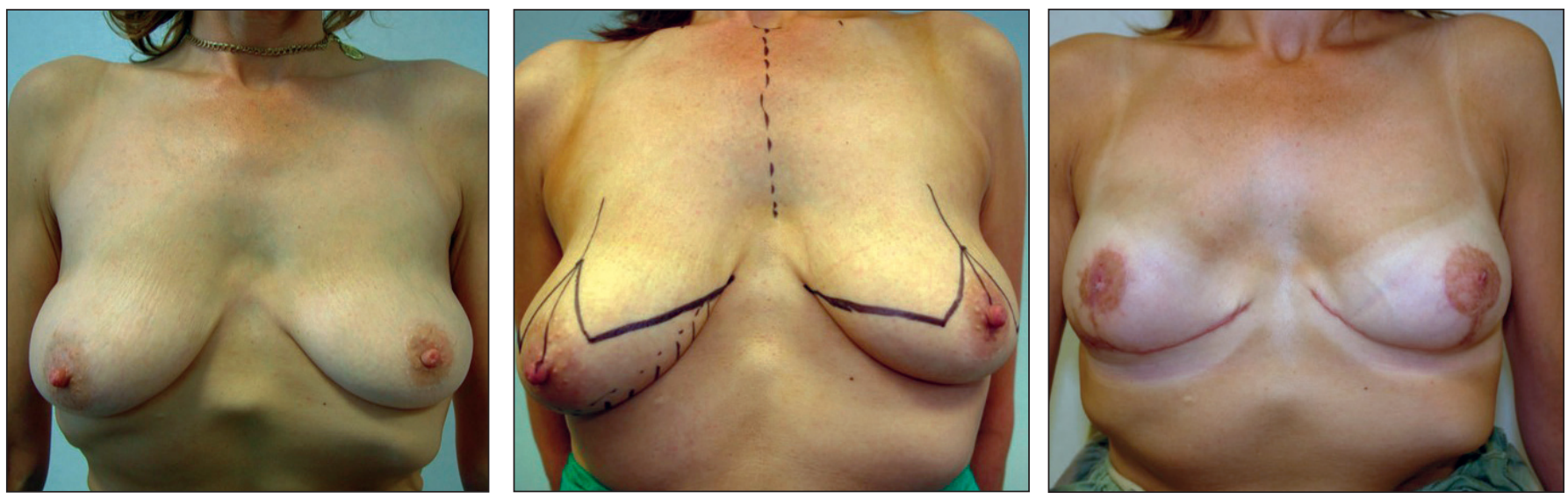

Figura 8. Caso 4. Indicación: cancerofobia. Paciente de 38 años con asimetría y tamaño moderado de las mamas. A y B. Preoperatorio. C. Postoperatorio a las 8 semanas. Implante anatómico de gel cohesivo de silicona de 250 cc.
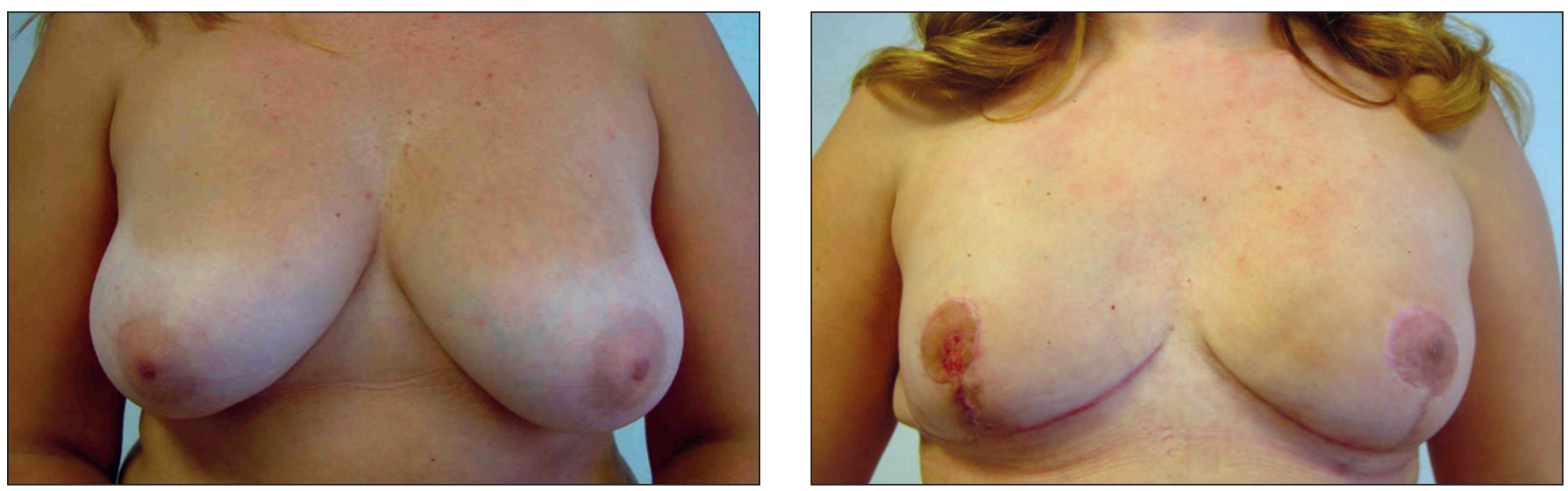

Figura 9. Caso 5. Indicación: antecedentes familiares. Paciente de 24 años con antecedente de madre y hermana con cáncer de mama y una tía materna con cáncer ovárico. A. Preoperatorio. B. Postoperatorio al mes. Implante anatómico de gel cohesivo de silicona de 250 cc.
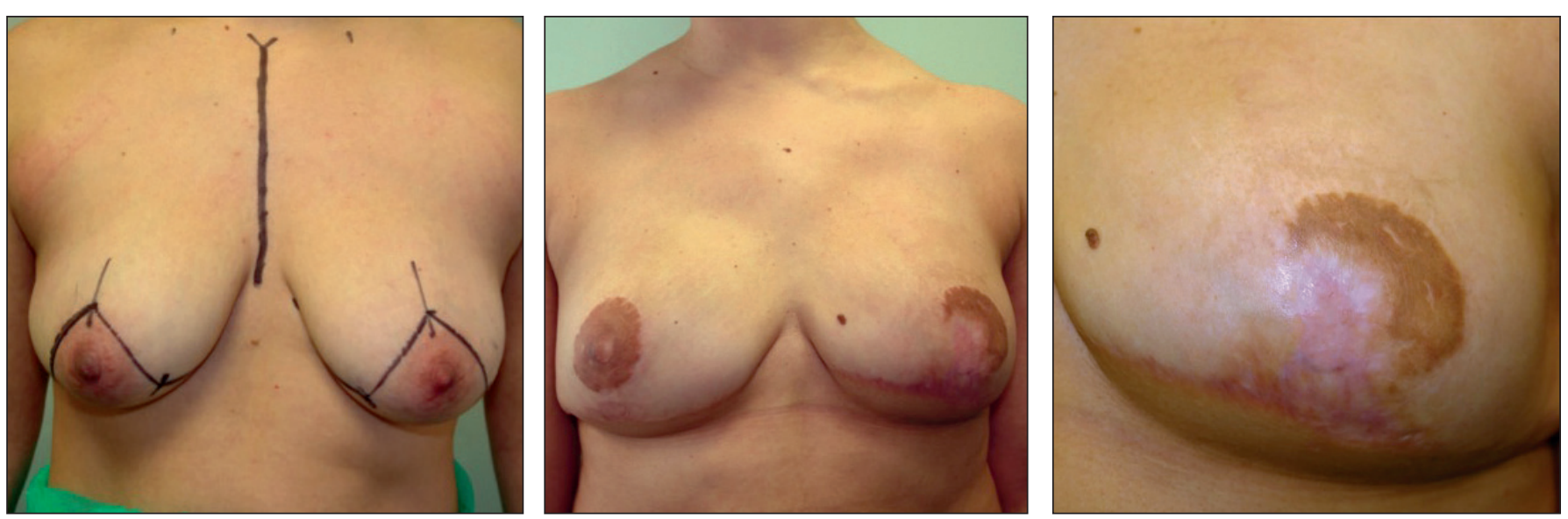

Figura 10. Caso 6. Indicación: Síndrome de Li Fraumeni. Paciente de 28 años de edad. Antecedente de osteosarcoma de miembro inferior izquierdo. A. Preoperatorio. B y C. Postoperatorio a los 6 meses. Implante anatómico de gel cohesivo de silicona de 265 cc.

Toth y col. ${ }^{(5)}$ describen en sus trabajos la mastectomía ahorradora de piel y dan un paso más proponiendo la reconstrucción inmediata en el mismo tiempo quirúrgico, disminuyendo así el tiempo en que las pacientes pueden verse mutiladas con la repercusión psicológica que esto conlleva.

En nuestro trabajo presentamos la mastectomía ahorradora de piel tipo IV con injerto libre del complejo areola-pezón (MIVAP) como una técnica de fácil aprendizaje, capaz de proporcionar cobertura adecuada de la prótesis mamaria y que evita la necesidad de colocar materiales extraños añadidos como matrices dérmicas ${ }^{(6)} \mathrm{o}$ de realizar diversos colgajos músculares para optimizar el espesor del tejido sobre la prótesis. ${ }^{(7)}$ Por otra parte, anclar el músculo pectoral al colgajo dermo-graso desepidermizado ubicado en el polo inferior, minimiza su retracción hacia arriba evitando la aparición de arrugas subcutáneas que pueden empeoran el resultado estético final.

Algunos estudios han encontrado que la mastectomía profiláctica de la mama sana podría mejorar la supervivencia. ${ }^{(8,9)} \mathrm{La}$ indicación más frecuente en nuestro trabajo fue el antecedente de cáncer ginecológico (ovario o 
mama) asociado a una mutación en el gen BRCA 1 o 2 , seguida por la positividad para mutación del BRCA 1 o 2 sin antecedentes tumorales y la cancerofobia.

Indicaciones como el síndrome de Li-Fraumeni, donde una mutación en el gen supresor p53 aumenta la susceptibilidad de presentar tumores como cáncer de mama, sarcomas de partes blandas, tumores cerebrales y osteosarcomas, ${ }^{(10)}$ son menos frecuentes. En este trabajo presentamos 1 caso en el que realizamos una MRR a una paciente con este síndrome.

En una serie de 18 casos en pacientes sometidas a mastectomía ahorradora de piel con reconstrucción inmediata utilizando implantes de silicona subpectorales con una técnica similar a la utilizada por nosotros, De1la Rovere y col. mencionan aproximadamente un 5\% de complicaciones como infección o hematoma y un porcentaje similar de pobre resultado cosmético a largo plazo producto de fibrosis en el polo inferior de la mama. ${ }^{(11)}$ En nuestra muestra, el pobre resultado estético se detectó en 2 pacientes $(22 \%)$. Según estos autores el principal problema que tienen las mastectomías clásicas, en las que no se da importancia al espesor del tejido entre el implante y la piel, viene dado por la falta de cobertura en el polo ínfero-medial. Por esta razón ellos prefieren, al igual que nosotros, la colocación de los implantes mamarios en el plano subpectoral, lo que sin duda mejora la cobertura.

Ninguna de nuestras pacientes presentó hematoma ni dehiscencia, pero en 1 caso fue necesario retirar una de las prótesis por una infección tardía de la zona quirúrgica. La fibrosis y la consiguiente retracción tisular cicatricial y pérdida de volumen en el polo ínfero-medial de la mama se presentó en diferentes grados. En todas ellas encontramos pérdida del espesor tisular secundario a fibrosis cicatricial, dando una imagen ancha al escote que a la vista impresiona como de una separación aumentada de las mamas.

El músculo se mantuvo en su sitio disminuyendo la tendencia a su retracción cefálica gracias a la sutura con el colgajo dermograso y en ningún caso se retrajo, proporcionando una cobertura correcta al polo superior de la mama. Creemos que la fibrosis inferior y el ensanchamiento del escote se debe a que el bolsillo protésico cede a la mecánica del tórax y al movimiento de los brazos, tendiendo a lateralizar los implantes.

Bajo la piel radiada existe mayor riesgo de infección e intolerancia a la prótesis, ${ }^{(12)}$ teniendo a veces que retirarlas para sustituirlas por otras o por tejido autólogo. Todas las pacientes de nuestra muestra toleraron adecuadamente las prótesis implantadas y ninguna había recibido radioterapia previa. Una paciente presentó linfedema grado IIa en relación con la BSGC.
Un 30\% de necrosis del CAP o del colgajo cutáneo es para Santanelli y col. el talón de Aquiles de este procedimiento. ${ }^{(13)}$ Esto produce una pérdida de volumen en el polo inferior de la mama y del CAP con un claro empobrecimiento del resultado estético. Solo 1 de nuestras pacientes presentó necrosis parcial del CAP que corregimos mediante la realización de un tatuaje definitivo con un color similar al resto de la areola; la paciente quedó satisfecha con el resultado logrado. Para disminuir el riesgo de presentar esta complicación, Wapnir y col. han descrito una técnica intraoperatoria en la que utilizan verde de indocianina para valorar mediante una cámara infrarroja computarizada el patrón vascular de la mama, lo que les permite controlar la disección durante toda la intervención, disminuyendo el riesgo de necrosis del $\operatorname{CAP}^{(14)}$ y/o del colgajo cutáneo. En nuestras pacientes no hemos utilizado técnicas como esta aunque creemos que podría resultar válido su empleo a la hora de evitar las complicaciones, claro está que a expensas de aumentar el coste de la intervención y el tiempo de quirófano. Hay que resaltar que este procedimiento solo se podría realizar en pacientes totalmente sanas y/o que no tengan ninguna indicación de extirpación del CAP por su proximidad con un tumor.

La MIVAP resulta especialmente útil en mujeres con glándulas mamarias grandes y la idea de realizar una MRR. Seis de las 9 pacientes de nuestra muestra deseaban además de la reducción del riesgo una reducción del tamaño de sus mamas, por lo que se beneficiaron de la mastectomía profiláctica y de la reducción y pexia mamarias.

Consideramos que, si se siguen adecuadamente los pasos de esta técnica, los resultados son reproducibles y la herida quirúrgica fácilmente cierra sin tensión y con una adecuada cobertura del implante por tejidos autólogos. De Vita y col. consideran este procedimiento como la técnica más adecuada para la reducción de riesgo de padecer cáncer mamario en pacientes con macromastia. ${ }^{(15)} \mathrm{La}$ MIVAP permite realizar la mastectomía reductora de riesgo, la reducción y la reconstrucción mamaria en un solo tiempo quirúrgico, cumpliendo adecuadamente con los 3 objetivos cuyo beneficio redunda en una menor cantidad de actos quirúrgicos y/o anestésicos, menos periodos de inactividad laboral y mejor recuperación de los tejidos tras una única intervención. ${ }^{(16)}$

\section{Conclusiones}

La MIVAP es una alternativa viable para proponer a las pacientes con mamas de tamaño moderado a grande que desean la realización de una MRR, y debe ser tenida en cuenta dentro del arsenal terapéutico en pacientes con alto riesgo de desarrollar un cáncer de mama. 
Realizar una mastectomía reductora de riesgo mediante la técnica descrita en este trabajo permite obtener un resultado satisfactorio en la mayoría de las pacientes siempre y cuando la indicación sea correcta. En general la valoración subjetiva y objetiva de los resultados globales de la técnica es percibida como favorable tanto por parte del equipo médico como de las pacientes.

La curva de aprendizaje de la MIVAP es corta. Tiene la ventaja de ser fiable y reproducible con resultados similares si se ejecuta meticulosamente. Solo es necesario un tiempo quirúrgico para la mastectomía y la reconstrucción, evitando así las complicaciones de un expansor cutáneo y la necesidad de una segunda intervención para su cambio por una prótesis definitiva.

\section{Dirección del autor}

Dr. Edgar Mauricio Avellaneda Oviedo

Servicio de Cirugía Plástica

Complejo Hospitalario Universitario de La Coruña

Avda. Las Xubias de Arriba s/n

15009 La Coruña, España.

\section{Bibliografía}

1. Tuttle TM., Habermann EB., Grund EH., Morris TJ., Virnig BA. Increasing use of contralateral prophylactic mastectomy for breast cancer patients: a trend toward more aggressive surgical treatment. J Clin Oncol Off J Am Soc Clin Oncol. 2007;25(33):5203-5209.

2. Lee TS., Morris CM., Czerniec SA., Mangion AJ. Does Lymphedema Severity Affect Quality of Life? Simple Question. Challenging Answers. Lymphat Res Biol. 2018;16(1):85-91.

3. Gómez-Campelo P., Bragado-Álvarez C., HernándezLloreda MJ. Psychological distress in women with breast and gynecological cancer treated with radical surgery. Psychooncology. 2014;23(4):459-466.

4. Ferlay J., Soerjomataram I., Dikshit R., Eser S., Mathers C., Rebelo M., et al. Cancer incidence and mortality worldwide: sources, methods and major patterns in GLOBOCAN 2012. Int $J$ Cancer J Int Cancer. 2015;136(5):E359-386.
5. Toth BA., Daane SP. Purse-string mastectomy with immediate prosthetic reconstruction: an improved skin-sparing technique for small breasts. Plast Reconstr Surg. 2003;111(7):2333-2337.

6. Aguilera-Sáez, J. et al. Experiencia en reconstrucción mamaria inmediata con implante y matriz acelular de pericardio bovino tras mastectomía ahorradora de piel. Cir. plást. iberolatinoam., 2015; 41(4):385-392.

7. Dávalos-Dávalos P., Ramírez-Rivera JI., Dávalos-Dávalos PA. Colgajos de pectoral mayor y oblicuo externo para cobertura de expansores y/o prótesis en reconstrucción postmastectomía. Cir. plást. iberolatinoam. 2015;41(1):33-39.

8. Peralta EA., Ellenhorn JD., Wagman LD., Dagis A., Andersen JS., Chu DZ. Contralateral prophylactic mastectomy improves the outcome of selected patients undergoing mastectomy for breast cancer. Am J Surg. 2000;180(6):439-445.

9. Boughey JC., Hoskin TL., Degnim AC., Sellers TA., Johnson JL., Kasner MJ., et al. Contralateral prophylactic mastectomy is associated with a survival advantage in high-risk women with a personal history of breast cancer. Ann Surg Oncol. 2010;17(10):2702-2709.

10. Vázquez Cajide I., Albaina Latorre L., Juaneda Gabelas M., Acea Nebril B., Antolín Novoa S. Cáncer de mama asociado a síndrome de Li-Fraumeni en dos hermanas. Rev Senol Patol Mamar Ed Impr. 2011;65-69.

11. della Rovere GQ., Nava M., Bonomi R., Catanuto G., Benson JR. Skin-reducing mastectomy with breast reconstruction and sub-pectoral implants. J Plast Reconstr Aesthetic Surg JPRAS. 2008;61(11):1303-1308.

12. Jagsi R., Jiang J., Momoh AO., Alderman A., Giordano SH., Buchholz TA., et al. Complications After Mastectomy and Immediate Breast Reconstruction for Breast Cancer: A ClaimsBased Analysis. Ann Surg. 2016;263(2):219-227.

13. Santanelli F., Longo B., Sorotos M., Farcomeni A., Paolini G. Flap survival of skin-sparing mastectomy type IV: a retrospective cohort study of 75 consecutive cases. Ann Surg Oncol. 2013;20(3):981-989.

14. Wapnir I., Dua M., Kieryn A., Paro J., Morrison .D, Kahn D., et al. Intraoperative imaging of nipple perfusion patterns and ischemic complications in nipple-sparing mastectomies. Ann Surg Oncol. 2014;21(1):100-106.

15. De Vita R., Pozzi M., Zoccali G., Costantini M., Gullo P., Buccheri EM., et al. Skin-reducing mastectomy and immediate breast reconstruction in patients with macromastia. J Exp Clin Cancer Res CR. 2015;34:120.

16. Colizzi L., Lazzeri D., Agostini T., Giannotti G., Ghilli M., Gandini D., et al. Skin-reducing mastectomy: new refinements. J Plast Surg Hand Surg. 2010;44(6):296-301. 


\title{
Comentario al artículo "Mastectomía ahorradora de piel tipo IV con injerto libre de areola- pezón para reconstrucción con prótesis en cirugía reductora de riesgo"
}

\author{
Cristian ERAZO \\ Cirujano Plástico, Hospital Clínico Universidad de Chile y Centro de la Mama Clínica Alemana, Santiago, Chile. \\ Director del Capítulo de Cirugía Mamaria de la FILACP 2018-2020.
}

En el presente trabajo, Avellaneda y col. nos describen una cohorte de pacientes sometidas a mastectomía de reducción de riego (MRR) por diferentes causas y reconstruidas mediante una técnica que combina conceptos ya conocidos de reconstrucción inmediata retropectoral con matriz dérmica acelular (MDA) e implantes con mastopexia. Sin embargo, lo novedoso de esta comunicación, es que en vez de usar una costosa MDA para la cobertura del polo inferior y lateral del implante, utilizan un delgado colgajo dermograso inferior desepeitelizado que bajo otras circunstancias se desecharía. Esto lo convierte en una excelente alternativa para abaratar costos y para ser utilizada en los países en los cuales las MDA no están ampliamente disponibles. Por otra parte, también amplían el rango de pacientes que pueden ser candidatas a MRR con conservación de complejo areola pezón y reconstrucción inmediata con implante, ya que para lograr un buen resultado estético en mamas grandes y ptósicas, ideales para esta nueva técnica, tradicionalmente se planteaba la remoción del complejo por el alto riego de necrosis al tener que movilizarlo pediculado al azar a su posición adecuada.

Para poder realizar esta técnica es fundamental que el equipo oncológico deje colgajos dermocutaneos bien vascularizados. Tal como fue mencionan en la discusión por los autores, la cámara de verde indiocianina, si está disponible, puede ayudar en los casos de duda de perfusión de los colgajos y sería recomendable para los que se inician en esta técnica contar siempre con expansores tisulares en quirófano para no estresar un colgajo con vascularización límite.

Otra ayuda la puede dar el análisis preoperatorio de la mamografía digital, en la cual el ojo entrenado puede estimar el grosor del tejido celular subcutáneo. En nuestra experiencia, pacientes con $1 \mathrm{~cm}$ o más de celular subcutáneo tendrían menor riesgo de complicaciones por necrosis de los colgajos dermocutáneos, situación que po- dría haber explicado el único caso de complicación por infección postquirúrgica tardía que requirió la remoción del implante.

Si bien se trata de una serie pequeña, el porcentaje de satisfacción desde el punto de vista del paciente medido mediante un sencillo PROM (patient-reported outcome measure) ad hoc fue bueno (7.4/10); sin embargo, hubiera sido interesante haber aplicado un PROM estandarizado y ampliamente usado como el módulo de reconstrucción mamaria del Breast $\mathrm{Q} 1^{(1)}$ para poder comparar sus resultados con otros trabajos a futuro.

Finalmente, sólo les planteo la pregunta de ¿por qué seguir utilizando implantes texturizados en pacientes con factores de riesgo para cáncer de mama o cancerofobia? Sabemos que en posición retromuscular no existen reales ventajas desde el punto de vista de contractura capsular con respecto al implante liso y sí existe un riesgo, aunque pequeño, de linfoma anaplásico de células gigantes asociado a implante mamario en los texturizados y un poco mayor cuando se utilizan en pacientes con cáncer de mama. Mi recomendación para este grupo de pacientes en particular, basado en la evidencia actual, ${ }^{(2)}$ seria utilizar implantes lisos.

No me queda más que felicitar a los autores por este novedoso y valioso aporte a al armamentario quirúrgico, con una aplicabilidad inmediata en nuestra realidad ibero-latinoamericana.

Bibliografía

1.- Pusic AL., Klassen AF., Scott AM., Klok JA., Cordeiro PG., Cano S. Development of a new patient-reported outcome measure for breast surgery: the BREAST-Q. J.Plast Reconstr Surg. 2009;124(2):345-355

2.- Collett DJ., Rakhorst H., Lennox P., Magnusson M., Cooter R., Deva A. Current Risk Estimate of Breast Implant-Associated Anaplastic Large Cell Lymphoma in Textured Breast Implants. Plast Reconstr Surg. 2019, 143(3S): 30S-40S. 


\section{Respuesta al comentario del Dr. Erazo}

\section{E. Mauricio AVELLANEDA OVIEDO}

Queremos agradecer de manera especial al Dr. Cristian Erazo por su crítica constructiva y todos los comentarios que realiza sobre nuestro trabajo. Para todo el grupo de autores es muy satisfactorio y resulta un aliciente para seguir compartiendo nuestra experiencia, darnos cuenta del impacto que puede tener nuestro trabajo en países hermanos.

Las técnicas que involucran el uso de matriz dérmica acelular (MDA), como bien lo indica el Dr. Erazo en su comentario, implican la necesidad de poseer cierto recurso económico (carencia que existe también en muchas zonas de países desarrollados y no solo de países en vías de desarrollo). "Reciclar" al máximo los valiosos tejidos vivos del propio paciente redundará en dos beneficios bien claros. El primero de ellos es minimizar la potencial respuesta inmune ante el material extraño con la consecuente rápida recuperación en el postoperatorio (en nuestro trabajo, la reincorporación al trabajo rondó los 34 días) y por otra parte, la eficiencia y efectividad que supone utilizar la propia materia prima autóloga, minimizando gastos al sistema de salud y reduciendo los días potencialmente perdidos por baja laboral. Creemos firmemente que realizar un injerto libre de areola-pezón (en los casos en los que no esté contraindicado), proporciona un aspecto más natural a la mama con respecto al que pudiera proporcionar otro tipo de injerto o un tatuaje. Cuando hablamos de mastectomía ahorradora de piel queremos llevar el término "ahorradora" a su máxima expresión.

En nuestro centro, el equipo funciona como una unidad integral de patología mamaria, por lo que normalmente el equipo oncológico deja colgajos adecuados y suficientemente vascularizados para este tipo de reconstrucción. Pese a ello, como bien menciona el Dr. Erazo en su comentario, es posible que el único caso que se presentó de infección postoperatoria fuera debido a un aporte sanguíneo insuficiente de los tejidos tras la mastectomía, sumado a un aumento del estrés tisular producto del edema y del volumen del implante mamario.

Las tecnologías de apoyo (mamografía digital, verde de indocianina, etc.) generalmente resultan favorables para optimizar el resultado y minimizar los riesgos de necrosis secundaria al sufrimiento vascular. Sin embargo, es necesario disponer de un centro de alto nivel que cuente con dicha tecnología para poder usarla. La utilización de una escala estandarizada, como el módulo de reconstrucción mamaria del Breast Q1, habría resultado muy apropiada. Esta debilidad será solventada cuando aumentemos nuestra muestra para estudios posteriores.

Respecto a la pregunta y comentario del Dr. Erazo de "¿por qué seguir utilizando implantes texturizados en pacientes con factores de riesgo para cáncer de mama o cancerofobia? Sabemos que en posición retromuscular no existen reales ventajas desde el punto de vista de contractura capsular con respecto al implante liso y sí existe un riesgo, aunque pequeño, de linfoma anaplásico de células gigantes (LACG) asociado a implante mamario en los texturizados y un poco mayor cuando se utilizan en pacientes con cáncer de mama. Mi recomendación para este grupo de pacientes en particular, basado en la evidencia actual, seria utilizar implantes lisos", decir por nuestra parte que, aunque resulte bajo el incremento del riesgo de desarrollar LACG en pacientes con antecedente de cáncer de mama cuando se usan implantes texturizados con respecto a la población general, actualmente estamos de acuerdo con su recomendación, contrastada con la reciente y actual evidencia científica la cual, dada su reciente publicación, no era del todo clara en el momento en el que se llevaron a cabo las intervenciones quirúrgicas de las pacientes recogidas en este trabajo. 